\title{
Short-to-medium range hydrologic forecast to manage water and agricultural resources in India
}

\author{
Reepal Shah ${ }^{1}$, A. K. Sahai ${ }^{2}$, Vimal Mishra ${ }^{1}$ \\ ${ }^{1}$ Civil Engineering, Indian Institute of Technology (IIT) Gandhinagar and ITRA Project: Measurement to \\ Management (M2M): Improved Water Use Efficiency and Agricultural Productivity through Experimental \\ Sensor Network. \\ ${ }^{2}$ Indian Institute of Tropical Meteorology (IITM) Pune, India \\ Correspondence to: Vimal Mishra (vmishra@iitgn.ac.in)
}




\section{Lead-7}
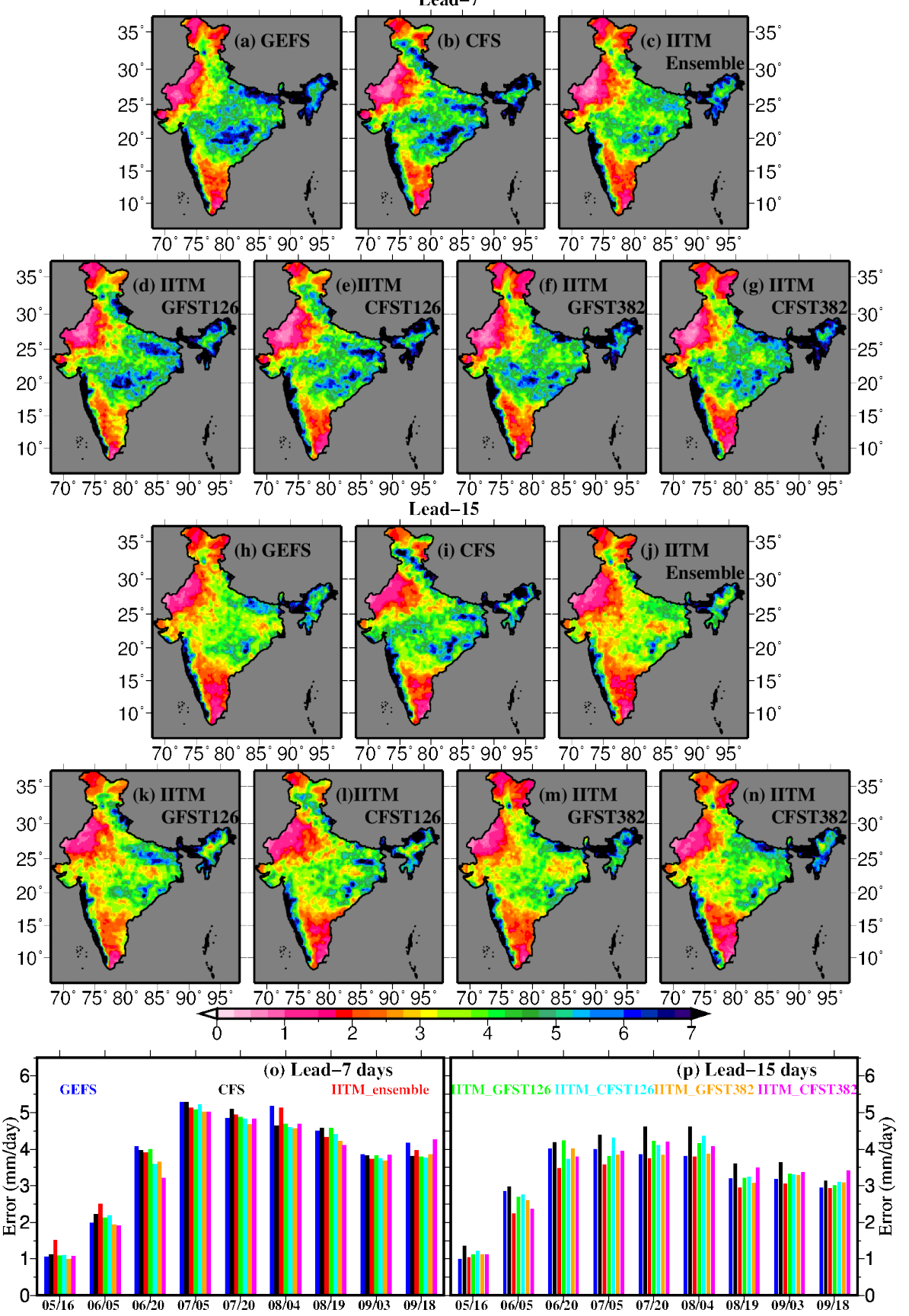

Figure S1: Median Absolute Error (MAE) in precipitation forecast with respect to the observed (OBS) precipitation. (a) Error in precipitation forecast from the GEFSv2 for 7-day lead, (b) same as (a) but for the CFSv2 (c) same as (a) but for the IITM- ensemble (d) same as (a) but for the IITM GFST126, (e) same as (a) but for the IITM CFST126, (f) same as (a) but for the IITMCFST382, (g) same as (a) but for the IITM GFST382. (h-n) same as (a-g) but for lead 15 day. (o) All India averaged MAE in different forecast dates at 7-day lead for forecast initiated on different dates in the monsoon season(p) same as (o) but for lead 15 day. Figure was prepared using GMTv4.5.9. 

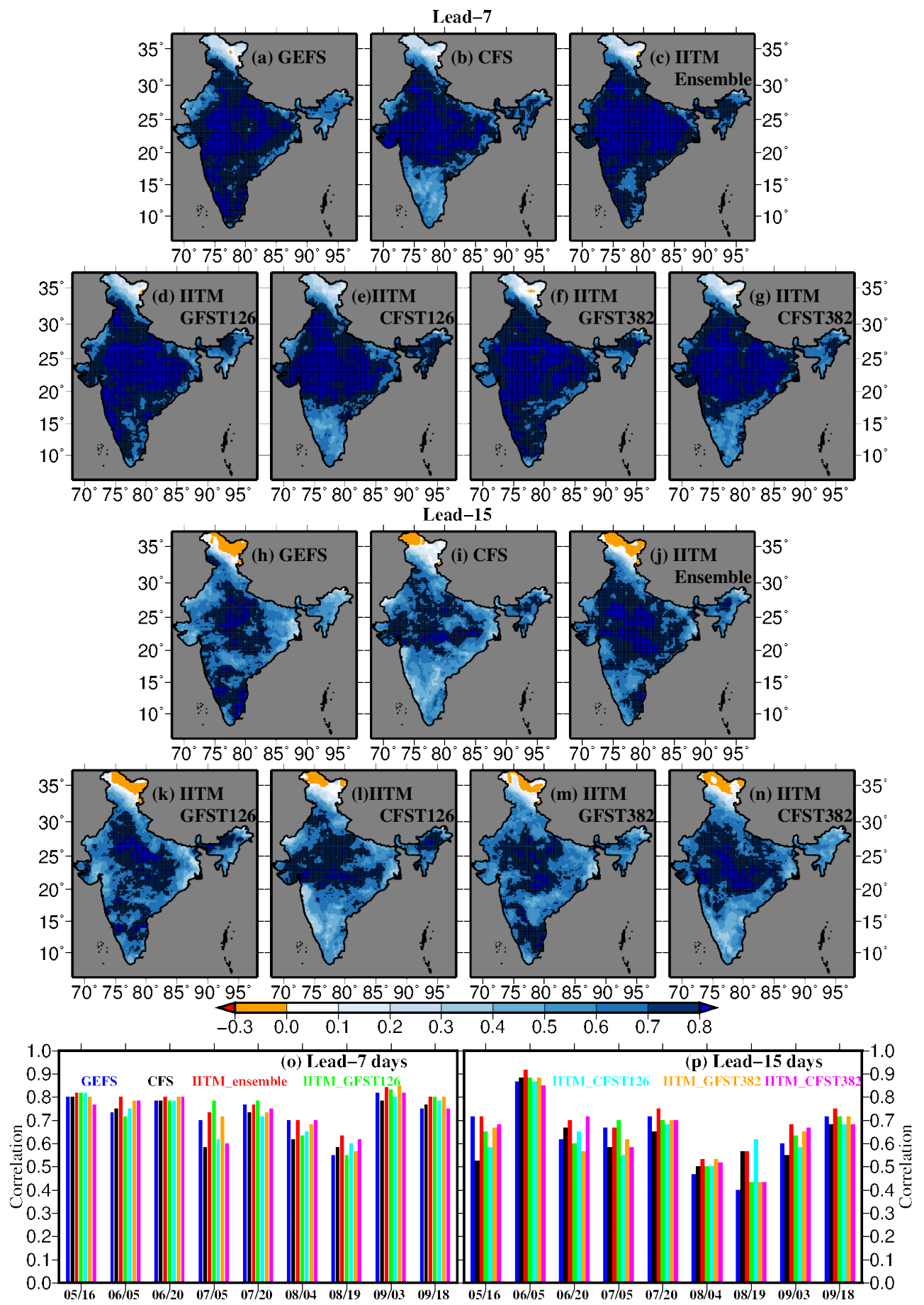

Figure S2: Correlation between daily maximum temperature (Tmax) forecast and OBS Tmax. (a) Correlation between Tmax forecast from the GEFSv2 and corresponding OBS at 7-day lead, (b-g) same as (a) but for the CFSv2, IITM ensemble, IITM GFST126, IITM CFST126, IITM GFST382, and IITM CFST382. (h-n) same as (a-g) but for lead 15 days. (o) All-India median correlation between different forecasts at 7-day lead and corresponding OBS for forecast initiated on different dates (h) same as (g) but for lead 15 days. Figure was prepared using GMTv4.5.9. 


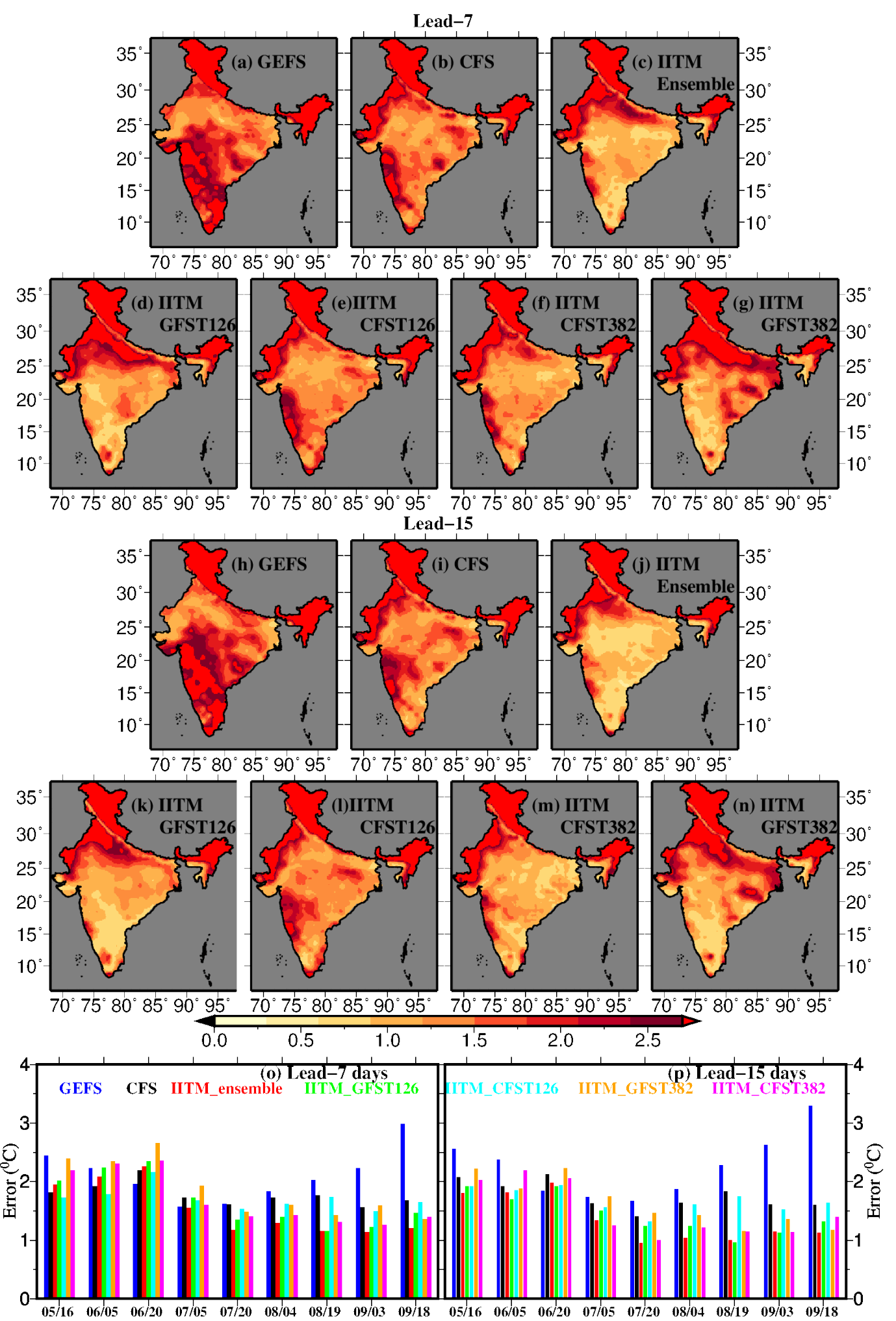

Figure S3: same as Figure S2 but for MAE. Figure wwas prepared using GMTv4.5.9. 
Lead-7
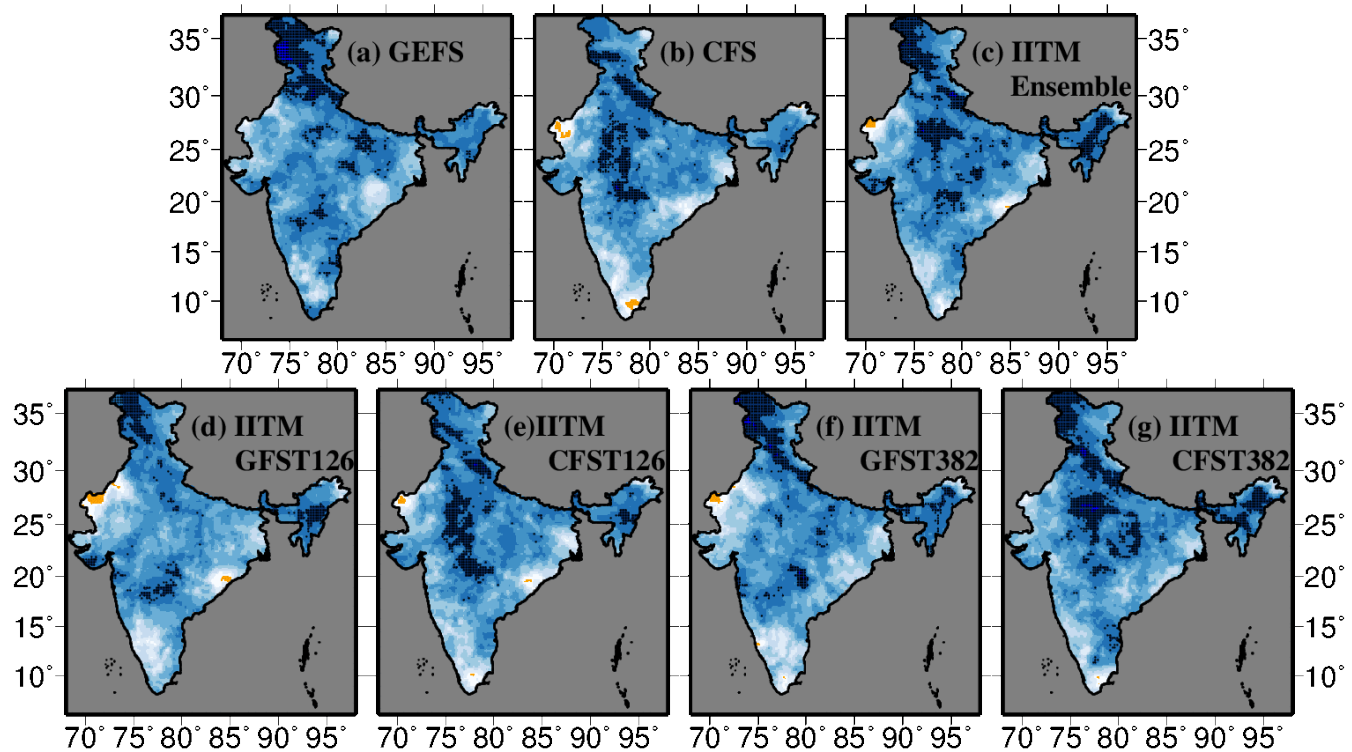

Lead-15
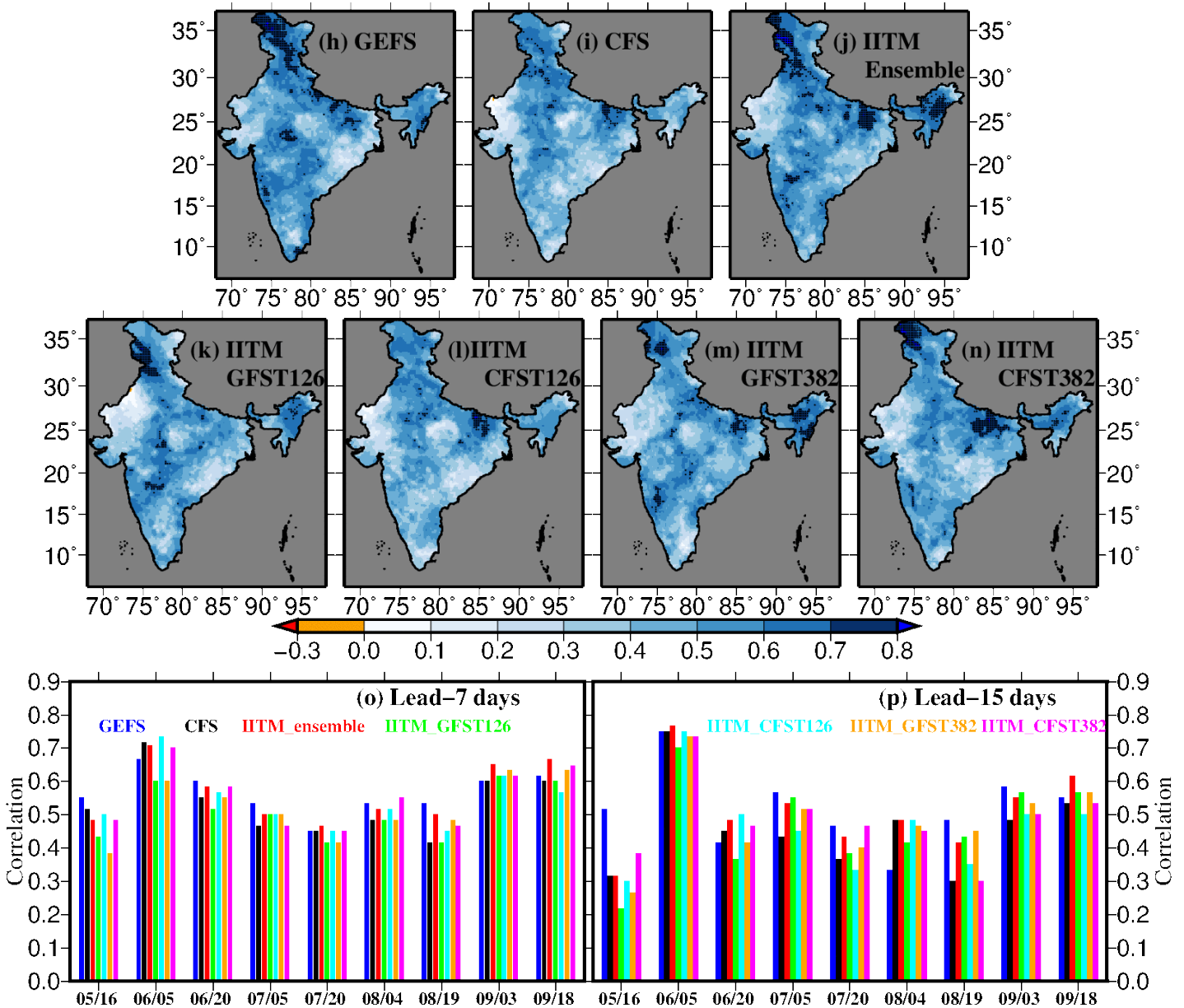

Figure S4: Same as Figure S2 but for minimum temperature (Tmin). Figure was prepared using GMTv4.5.9. 


\section{Lead-7}
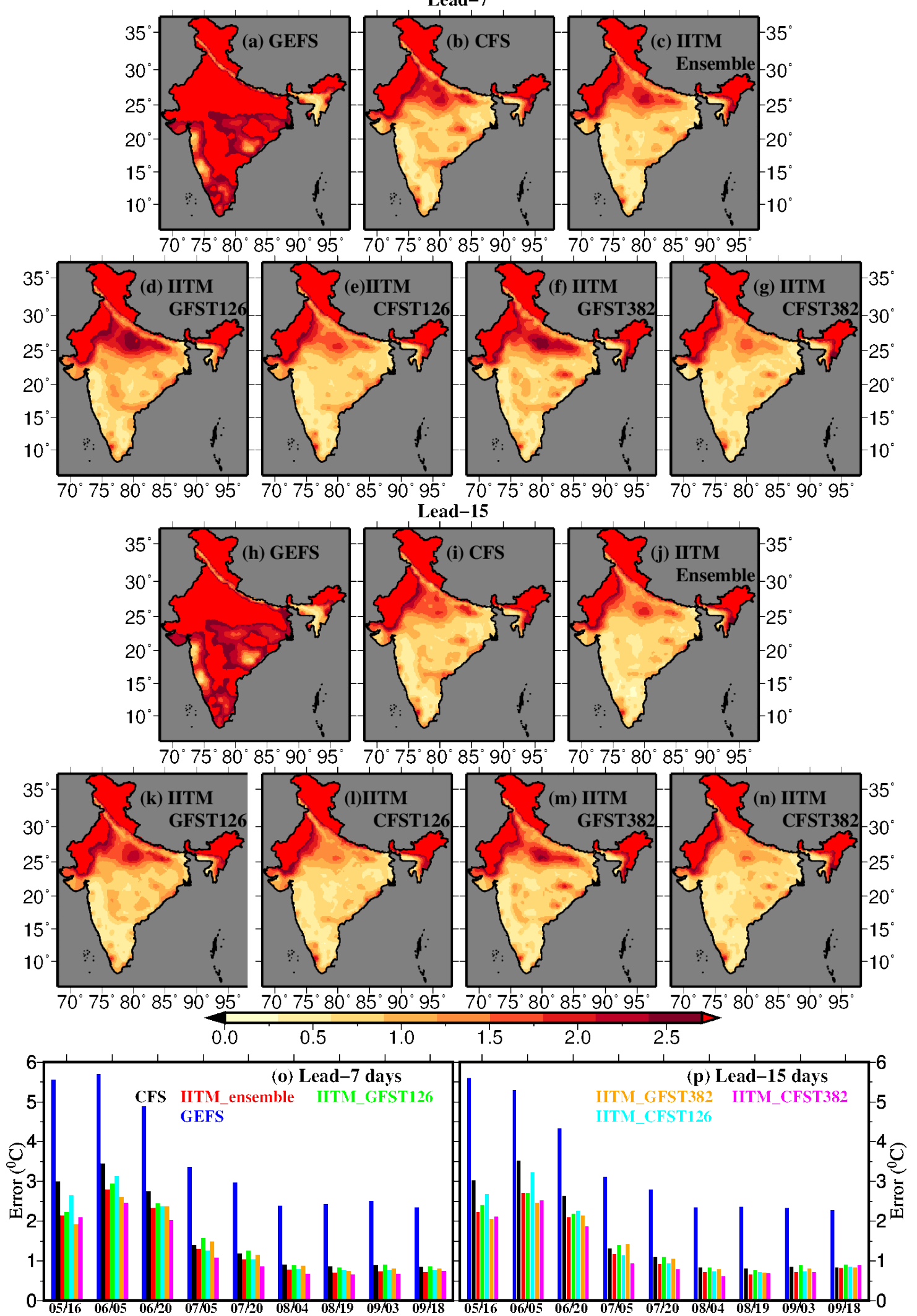

Figure S5: Same as Figure S3 but shows MAE in Tmin. Figure was prepared using GMTv4.5.9. 


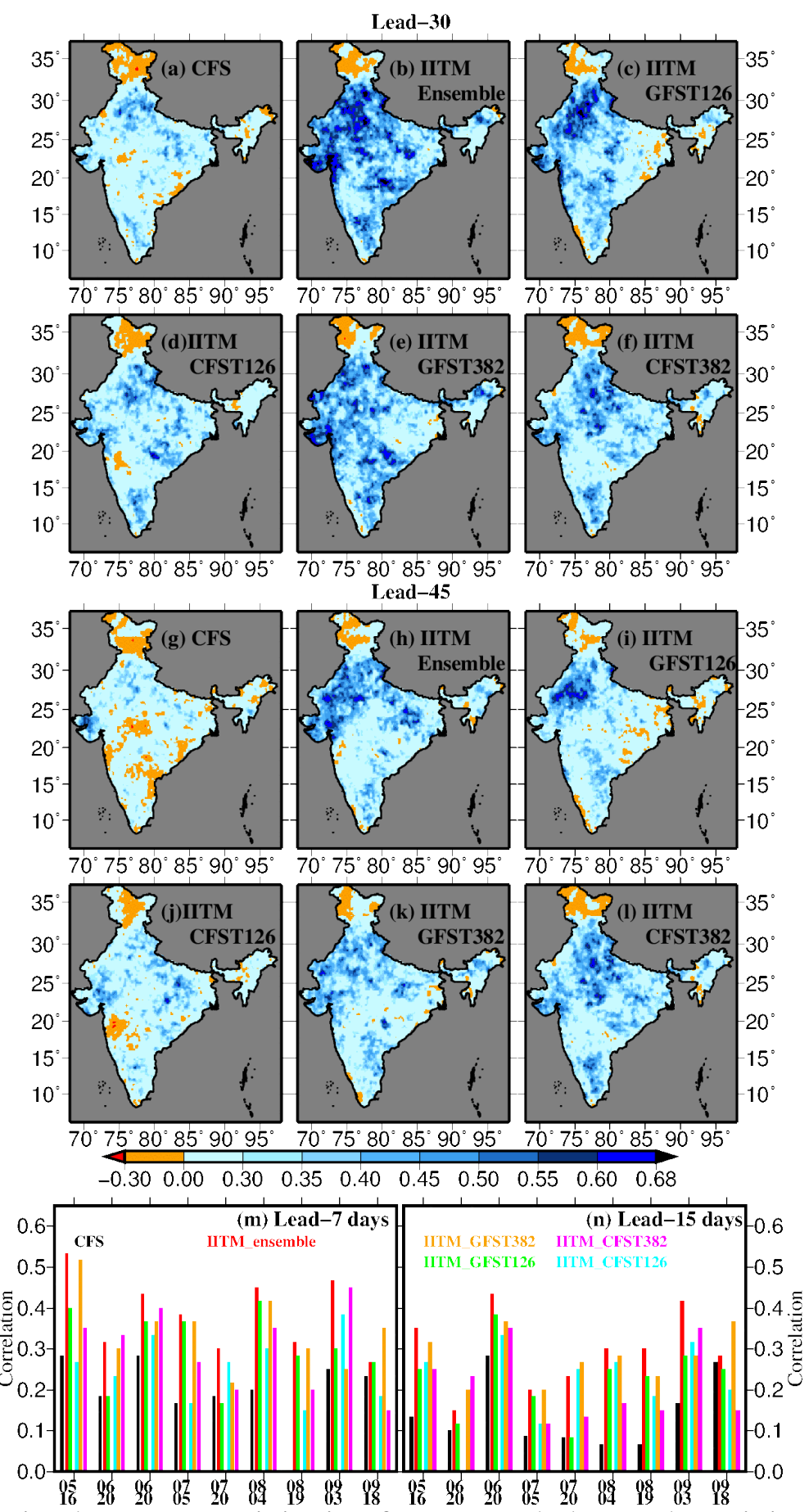

Figure S6: Correlation between precipitation forecast and observed precipitation (OBS). (a) Correlation between precipitation forecast from the CFS2v2 at 30-day and corresponding OBS, (b-f) same as (a) but for the IITM ensemble, IITM GFST126, IITM CFST126, IITM GFST382, and IITM CFST382, respectively. (g-l) same as (a-f).) but for lead-45 days. (m) All-India median correlation between different forecast dates and corresponding OBS for forecast initiated on different dates for 30 day lead (n) same as (m) but for 45 days lead. Figure was prepared using GMTv4.5.9. 

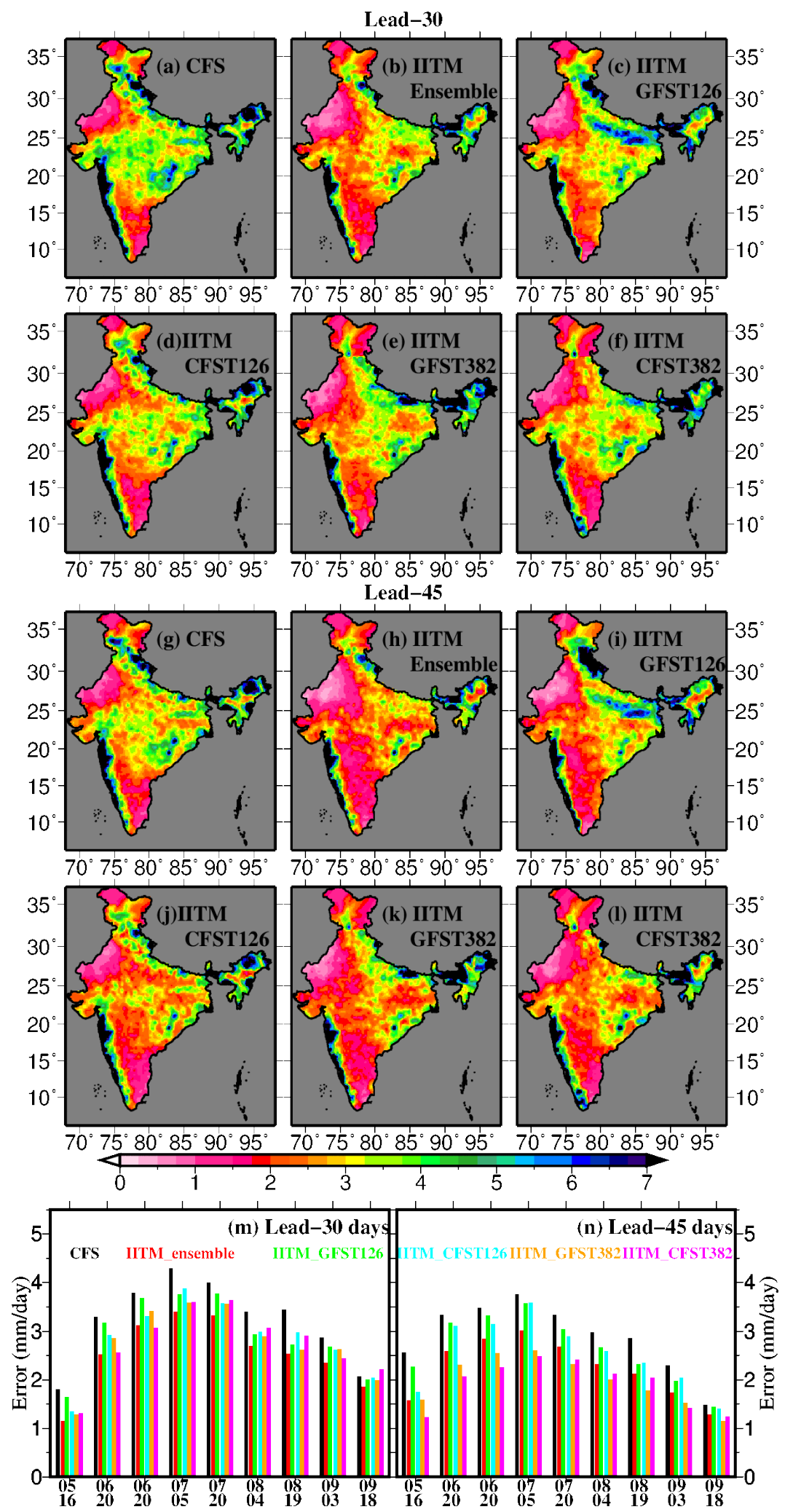

Figure S7: Same as Fig.S6 but for MAE. Figure was prepared using GMTv4.5.9. 


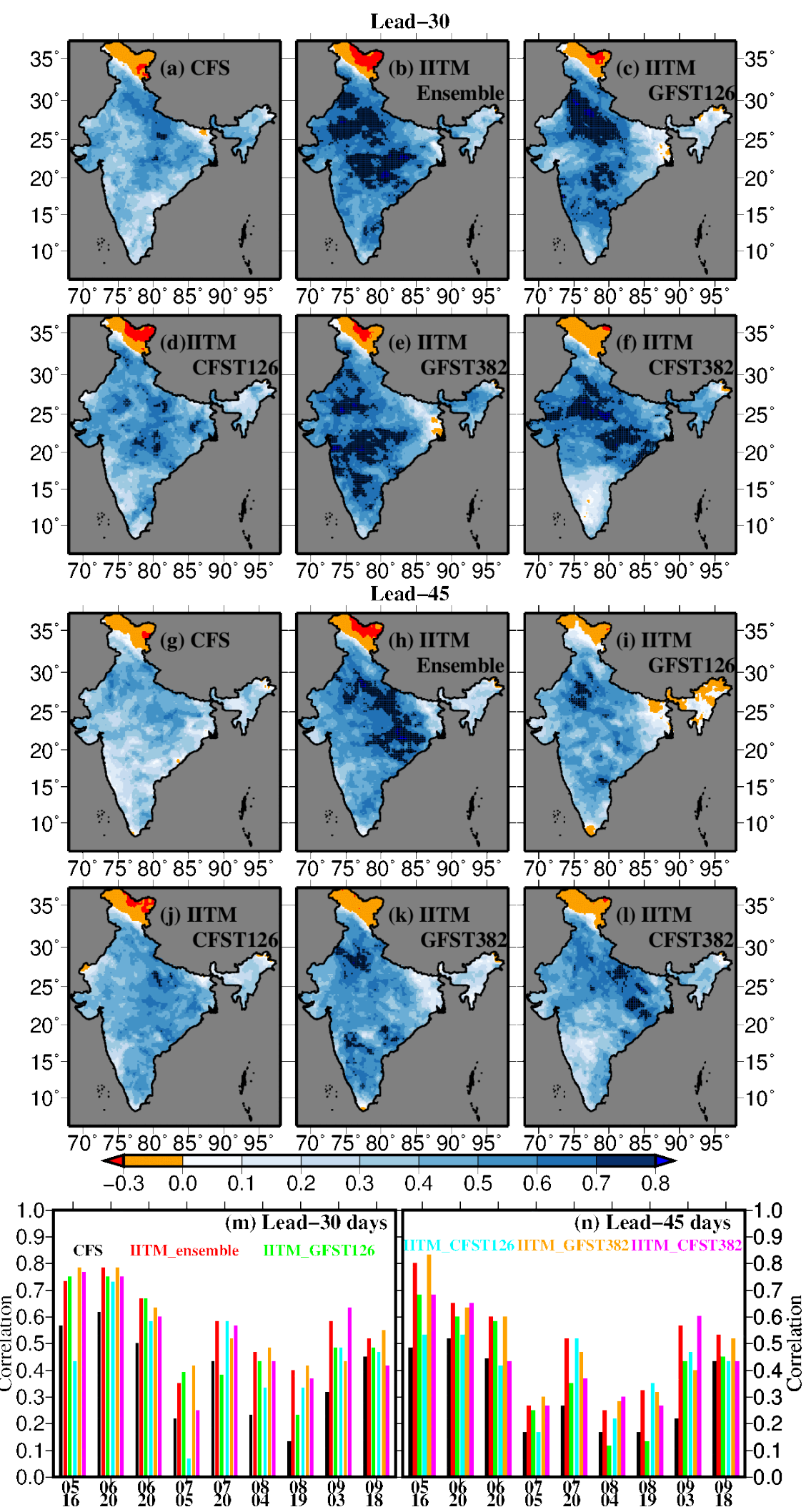

Figure S8: Same as Fig. S6 but for Tmax. Figure was prepared using GMTv4.5.9. 

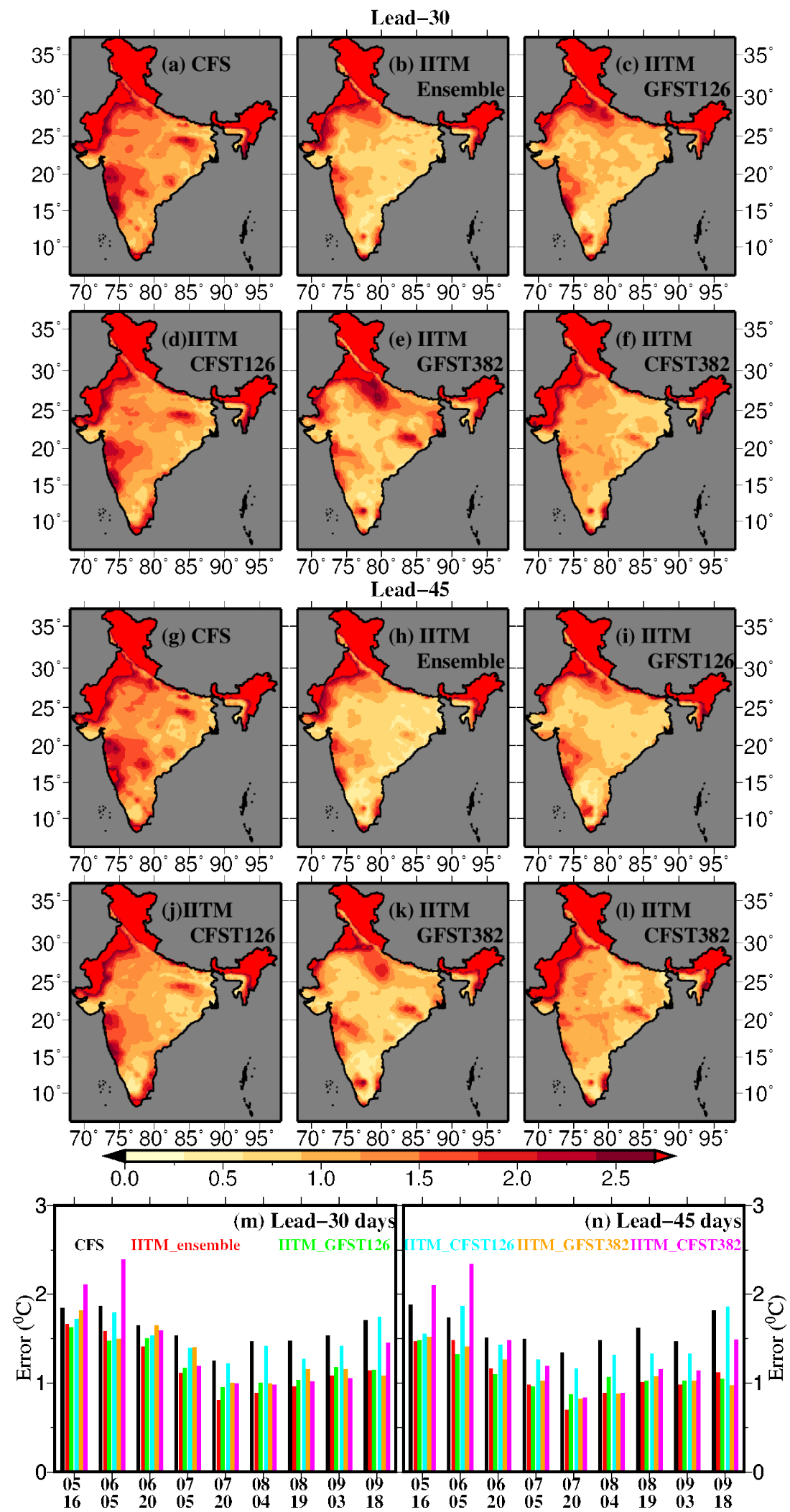

Figure S9: Same as Figure S6 but for MAE in Tmax. Figure was prepared using GMTv4.5.9. 


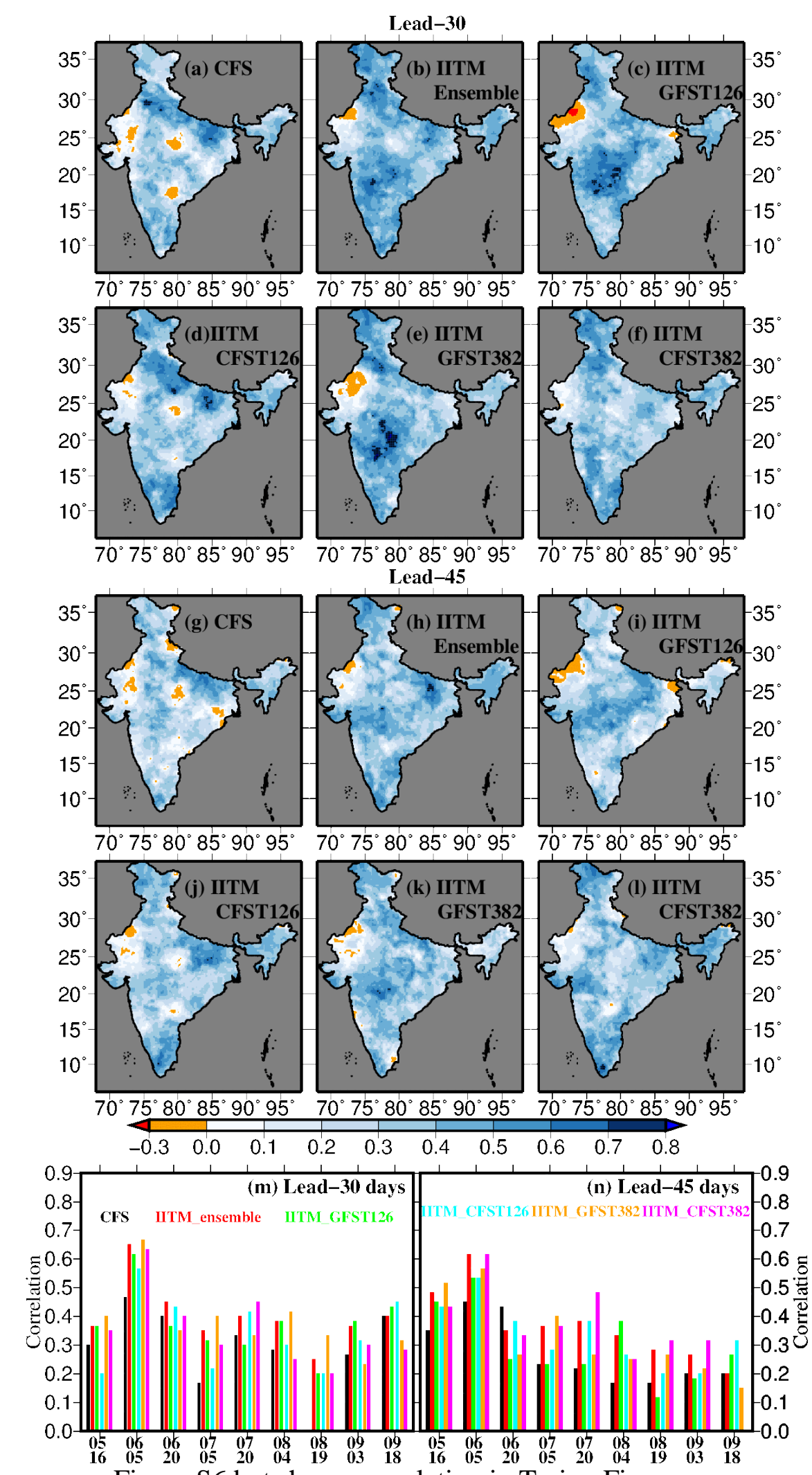

Figure S10: same as Figure S6 but shows correlation in Tmin. Figure was prepared using GMTv4.5.9. 


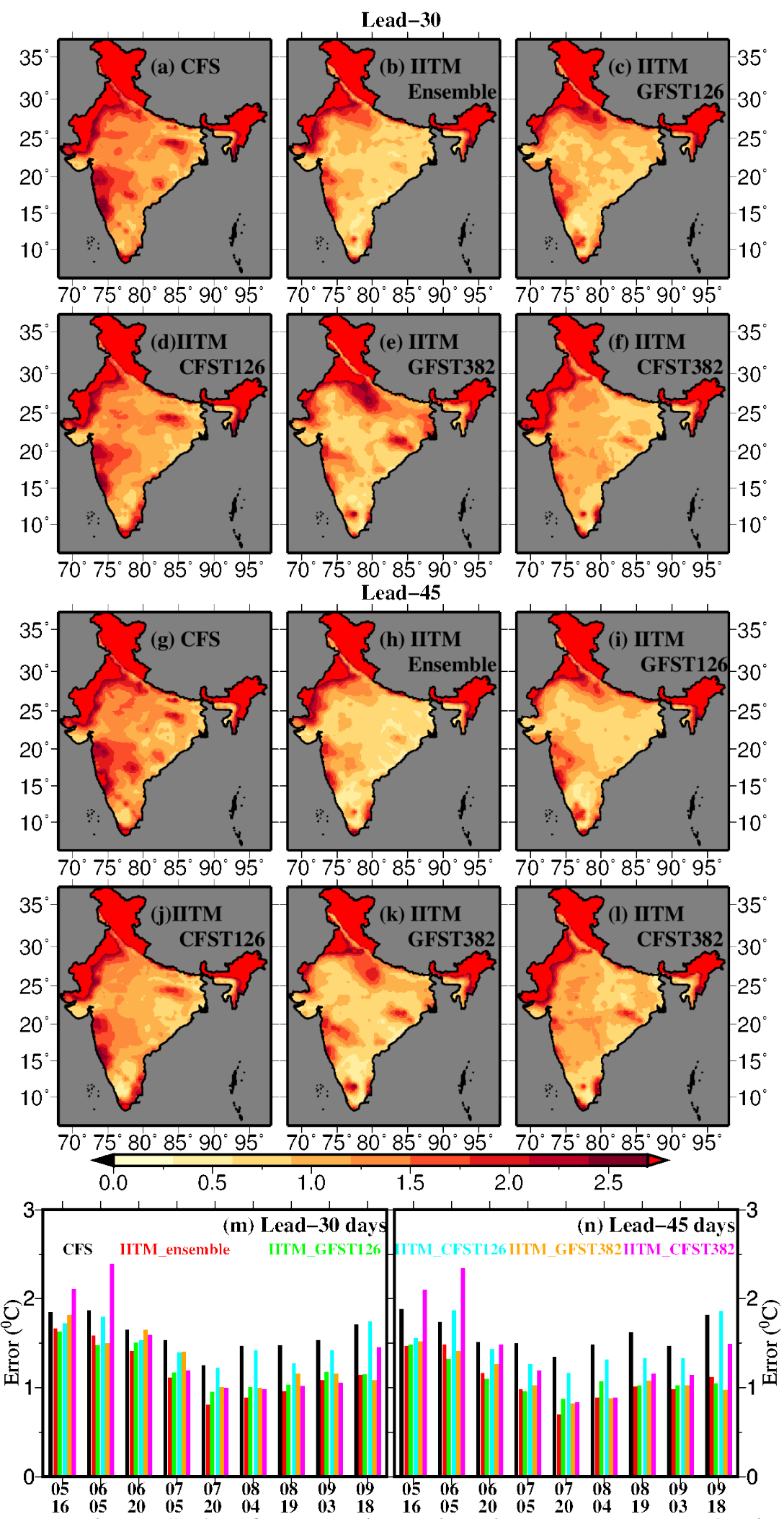

Figure S11: same as Figure S6 but for MAE in Tmin. Figure was prepared using GMTv4.5.9. 


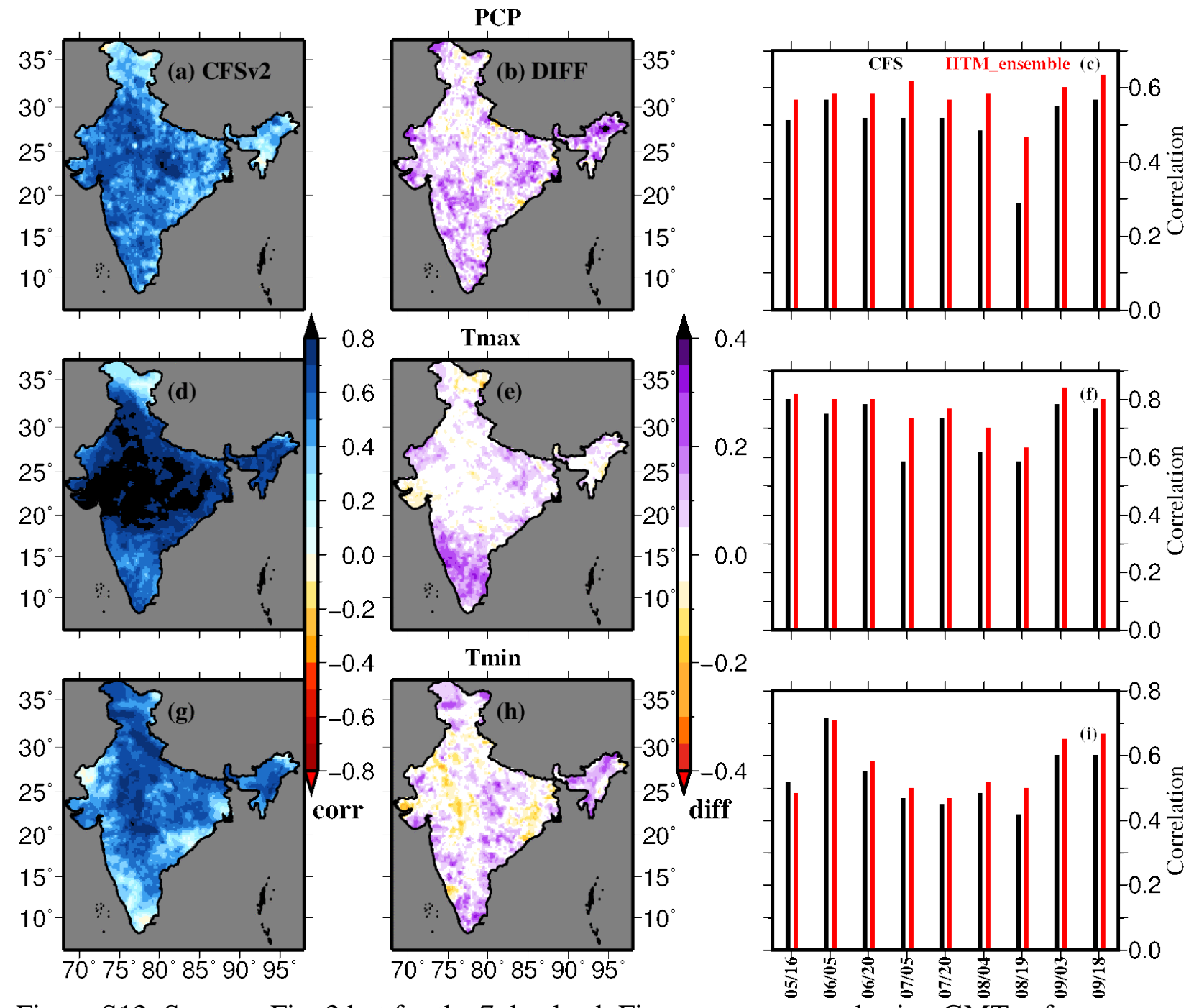

Figure S12: Same as Fig. 2 but for the 7 day lead. Figure was prepared using GMT software. 


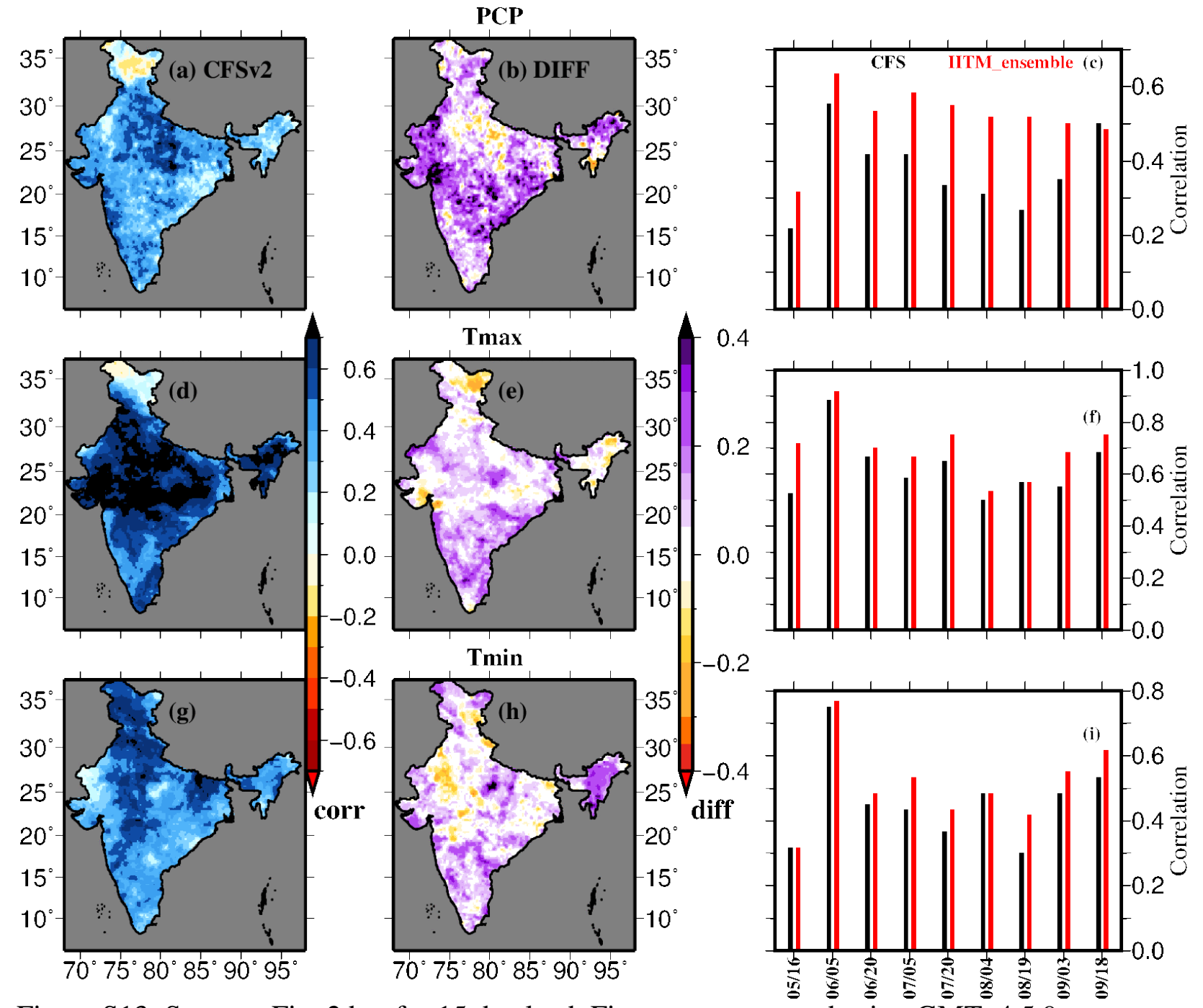

Figure S13: Same as Fig. 2 but for 15 day lead. Figure was prepared using GMTv4.5.9. 


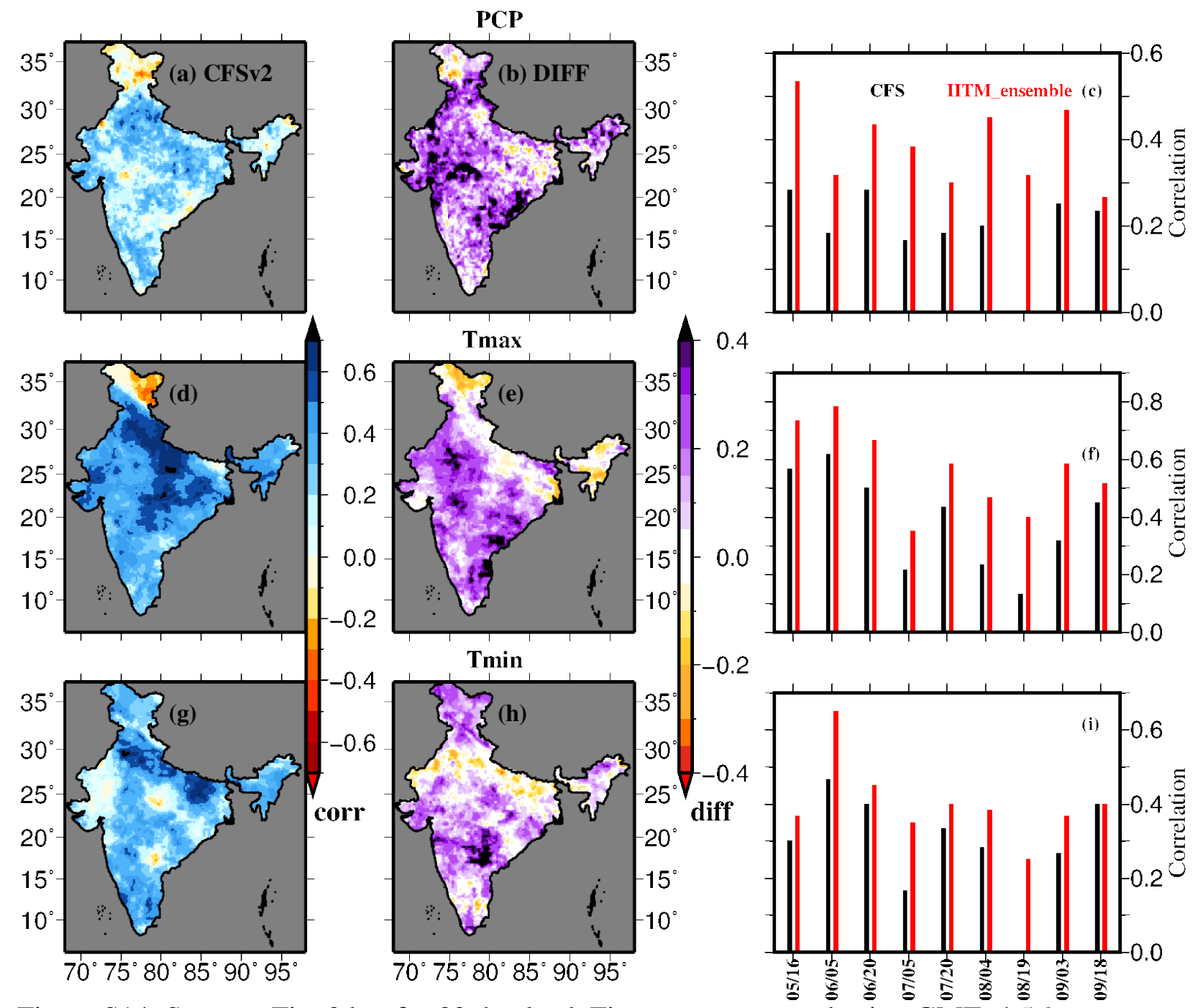

Figure S14: Same as Fig. 2 but for 30 day lead. Figure was prepared using GMTv4.5.9. 

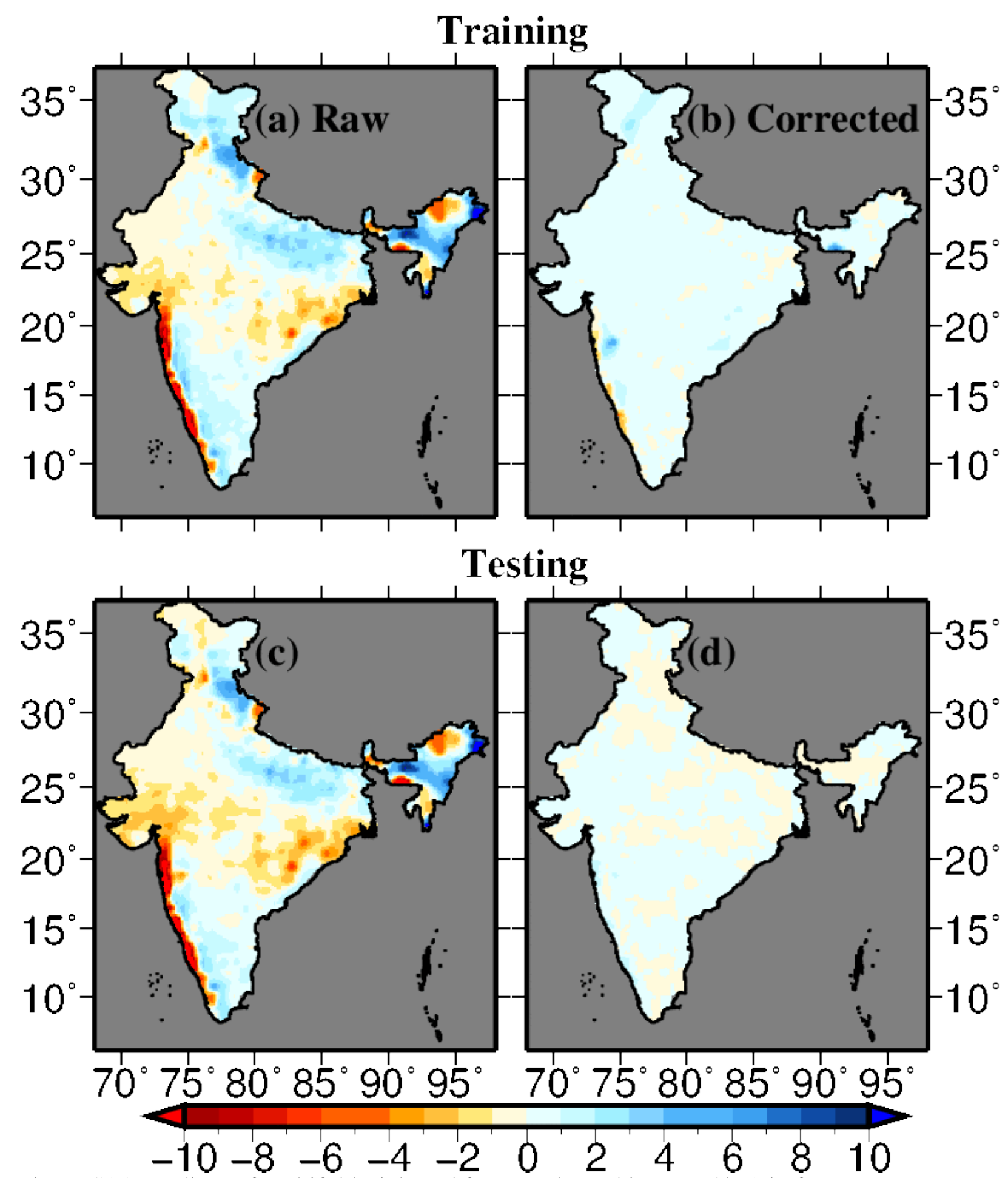

Figure S15: Median (of multifold trials and forecast dates) bias (mm/day) in forecast (accumulated for lead up to 45 days) from the IITM-ensemble before and after the bias correction. ( $\mathrm{a}$ and $\mathrm{b}$ ) Median bias ( $\mathrm{mm} /$ day) in precipitation forecast before and after correction, respectively during training years (9 years). (c and d) same as (a and b) but for testing year (1 year). Figure was prepared using GMTv4.5.9. 


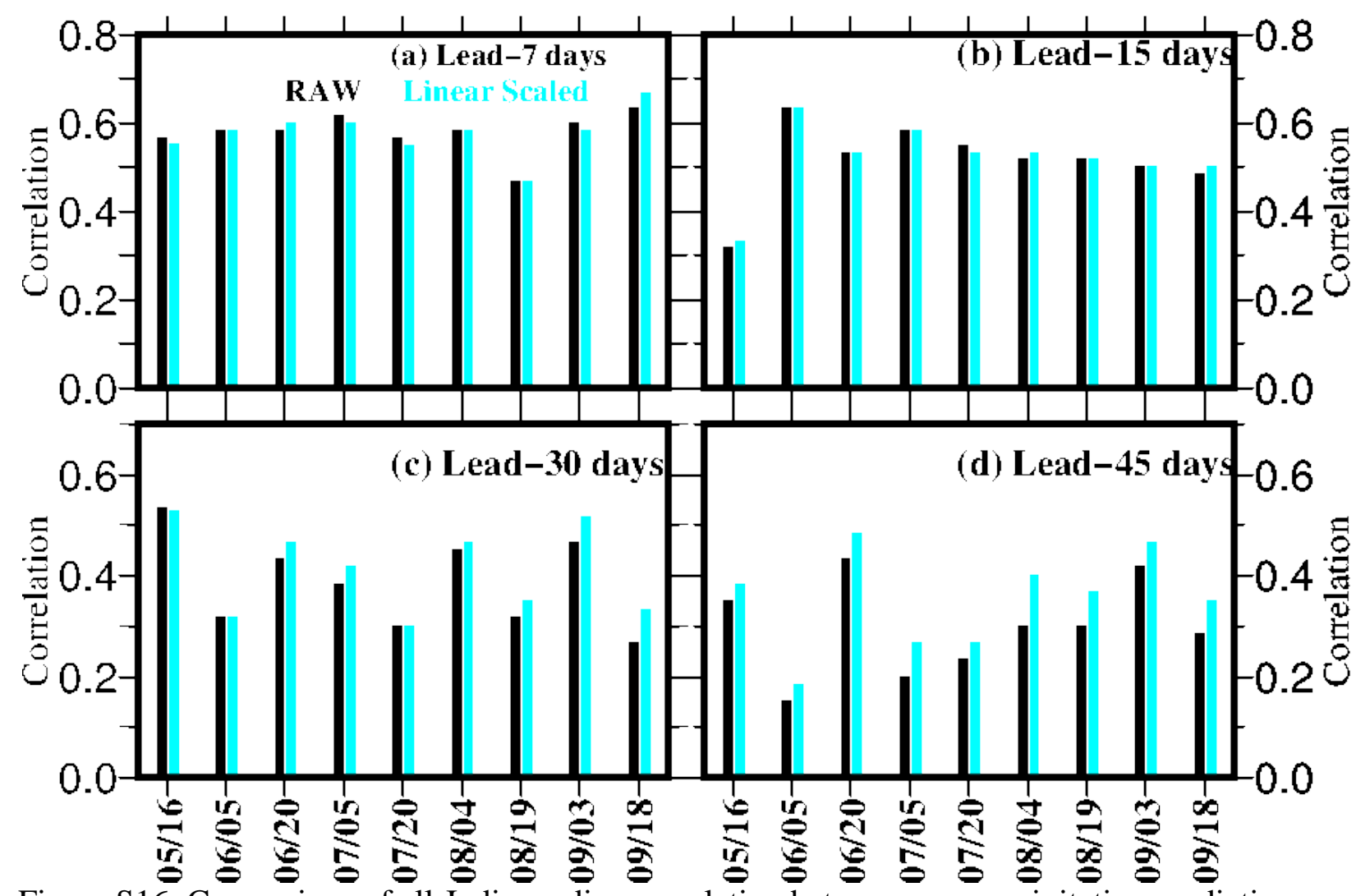

Figure S16: Comparison of all-India median correlation between raw precipitation prediction from the IITM-ensemble and OBS (cyan color) and bias-corrected precipitation prediction from IITM-ensemble and OBS (black color) for different forecast dates during the monsoon season and at different leads. Figure was prepared using GMTv4.5.9. 

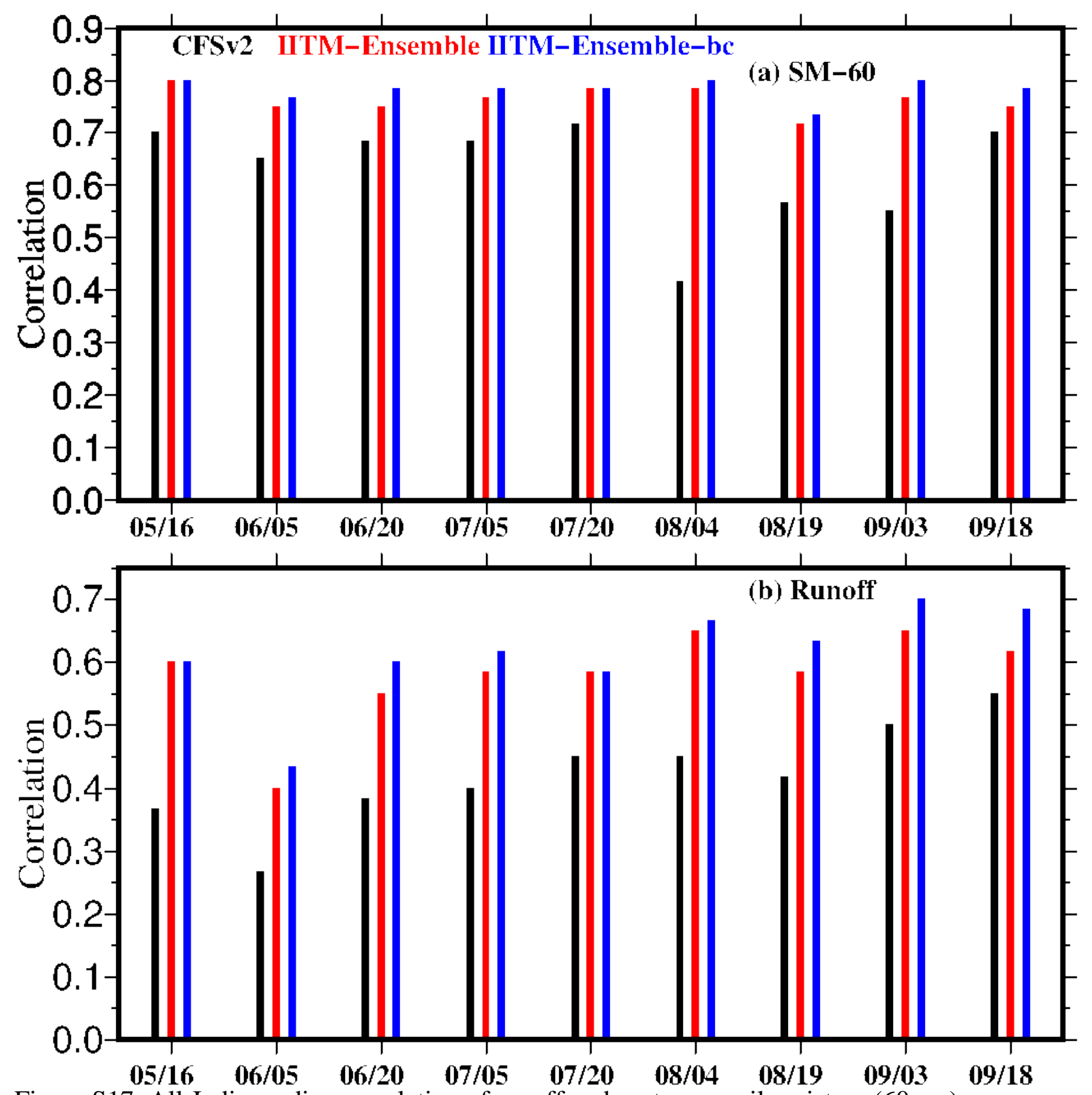

Figure S17: All-India median correlation of runoff and root zone soil moisture $(60 \mathrm{~cm})$ predicted using the VIC model and forecast with that simulated using the VIC model and OBS forcings for forecast dates during the monsoon season for the period of 2000-2009. Figure was prepared using GMTv4.5.9. 

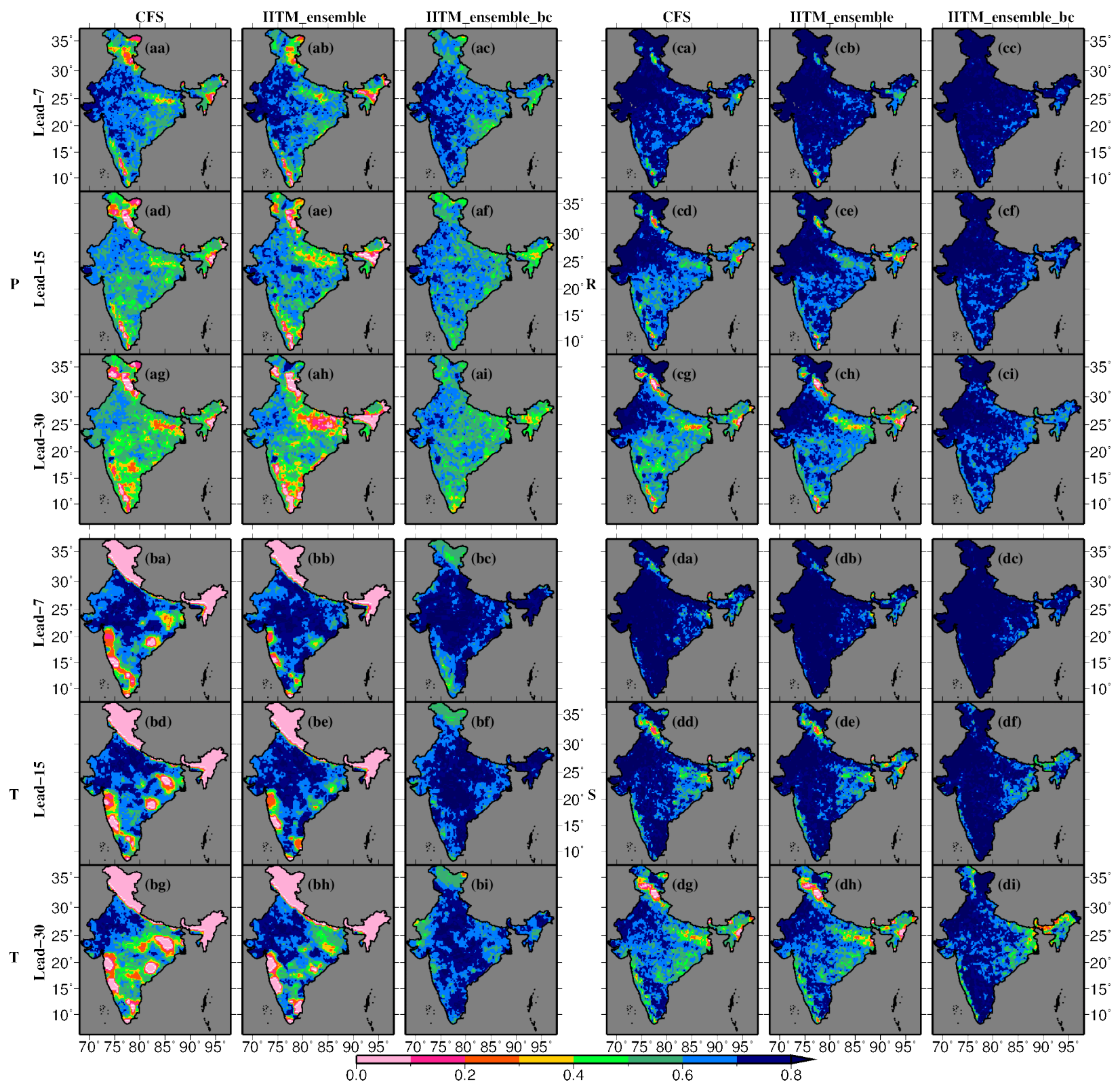

Figure S18: Critical Success Index (CSI, averaged for forecast dates) of predicting anomalies with respect to observed anomalies. Anomalies for particular year were predicted with respect to observed climatology. (aa,ad,ag) shows precipitation anomalies predicted by the CFSv2 (ab,ae,ah), IITM-ensemble, and (ac,af,ai) by bias-corrected IITM-ensemble for lead7,15 , and 30 respectively. (ba-bi) same as (aa-af) but for (for lead time) temperature anomalies. (ca-ci) same as (aa-ai) but for runoff anomalies. (da-di) same as (aa-ai) but for the root zone soil moisture. Figure was prepared using GMTv4.5.9. 

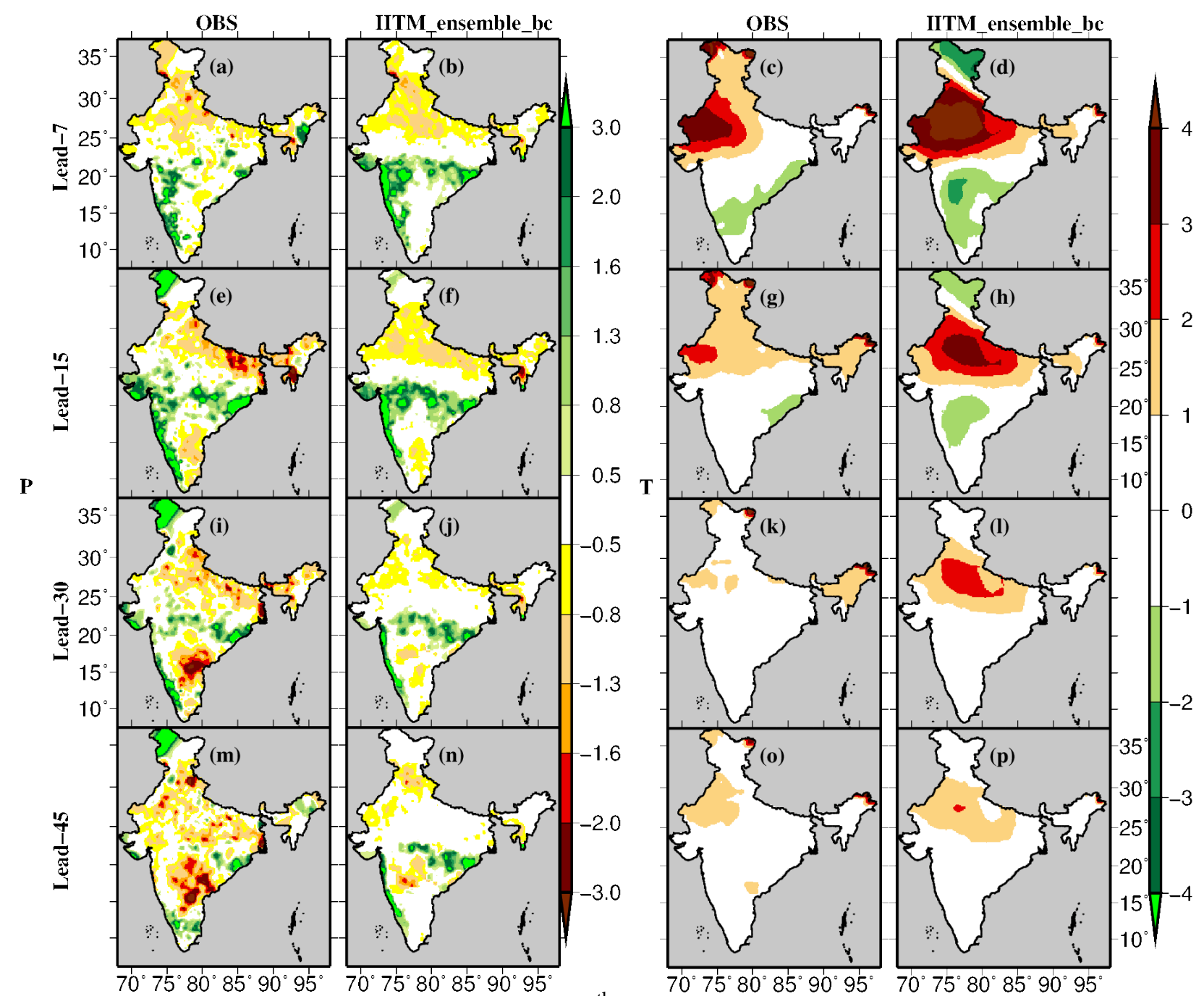

Figure S19: Forecast of anomalies initiated on $5^{\text {th }}$ July, 2009 for lead 7, 15, 30, and 45 days of meteorological variables. (a) Observed (standardized) anomalies in precipitation runoff at lead-7 days (a) anomalies in forecasted precipitation using IITM-ensmeble for lead-7 days. (c and d) same as (a and b) but shows anomalies in temperature for 7 days lead. (e-h), (i-l), (mp) same as (a-b) but for 15, 30, and 45 days lead. Figure was prepared using GMTv4.5.9. 
Table S1: Comparison of median skills (of considered forecast dates and of all-India) of different predicted variables for different forecast products.

\begin{tabular}{|c|c|c|c|c|c|c|c|c|c|}
\hline & & $\begin{array}{l}\text { le } \\
\text { ad }\end{array}$ & $\begin{array}{l}\text { GEF } \\
\text { Sv2 }\end{array}$ & $\begin{array}{l}\text { CF } \\
\text { Sv2 }\end{array}$ & $\begin{array}{l}\text { IITM_en } \\
\text { semble }\end{array}$ & $\begin{array}{l}\text { IITM_G } \\
\text { FST126 }\end{array}$ & $\begin{array}{l}\text { IITM_C } \\
\text { FST126 }\end{array}$ & $\begin{array}{l}\text { IITM_G } \\
\text { FST382 }\end{array}$ & $\begin{array}{l}\text { IITM_C } \\
\text { FST382 }\end{array}$ \\
\hline \multirow{8}{*}{$\begin{array}{l}\text { Precipi } \\
\text { tation }\end{array}$} & \multirow{4}{*}{$\begin{array}{l}\text { Correlat } \\
\text { ion }\end{array}$} & 7 & 0.55 & 0.52 & 0.58 & 0.55 & 0.53 & 0.57 & 0.57 \\
\hline & & 15 & 0.50 & 0.35 & 0.52 & 0.43 & 0.42 & 0.47 & 0.47 \\
\hline & & 30 & & 0.20 & 0.38 & 0.30 & 0.27 & 0.35 & 0.33 \\
\hline & & 45 & & 0.10 & 0.30 & 0.25 & 0.25 & 0.28 & 0.17 \\
\hline & \multirow{4}{*}{$\begin{array}{l}\text { MAE(m } \\
\text { m/day) }\end{array}$} & 7 & 2.86 & 2.50 & 2.62 & 4.00 & 3.77 & 2.55 & 2.85 \\
\hline & & 15 & 2.62 & 2.82 & 2.36 & 3.32 & 3.30 & 2.53 & 2.77 \\
\hline & & 30 & & 2.70 & 2.10 & 2.93 & 2.97 & 2.32 & 2.34 \\
\hline & & 45 & & 2.39 & 1.90 & 2.66 & 2.58 & 1.99 & 2.07 \\
\hline \multirow{8}{*}{ Tmax } & \multirow{4}{*}{$\begin{array}{l}\text { Correlat } \\
\text { ion }\end{array}$} & 7 & 0.75 & 0.75 & 0.80 & 0.78 & 0.75 & 0.78 & 0.75 \\
\hline & & 15 & 0.67 & 0.58 & 0.70 & 0.65 & 0.62 & 0.65 & 0.68 \\
\hline & & 30 & & 0.43 & 0.58 & 0.48 & 0.47 & 0.52 & 0.57 \\
\hline & & 45 & & 0.27 & 0.53 & 0.43 & 0.43 & 0.47 & 0.43 \\
\hline & \multirow{4}{*}{$\begin{array}{l}\text { MAE } \\
\left({ }^{\circ} \mathbf{C}\right)\end{array}$} & 7 & 2.0 & 1.7 & 1.3 & 1.5 & 1.7 & 1.6 & 1.4 \\
\hline & & 15 & 2.3 & 1.6 & 1.2 & 1.3 & 1.6 & 1.5 & 1.3 \\
\hline & & 30 & & 1.5 & 1.1 & 1.2 & 1.4 & 1.2 & 1.2 \\
\hline & & 45 & & 1.5 & 1.0 & 1.1 & 1.3 & 1.0 & 1.2 \\
\hline \multirow{8}{*}{ Tmin } & \multirow{4}{*}{$\begin{array}{l}\text { Correlat } \\
\text { ion }\end{array}$} & 7 & 0.55 & 0.52 & 0.52 & 0.50 & 0.52 & 0.50 & 0.55 \\
\hline & & 15 & 0.52 & 0.45 & 0.48 & 0.43 & 0.48 & 0.47 & 0.47 \\
\hline & & 30 & & 0.30 & 0.38 & 0.37 & 0.32 & 035 & 0.30 \\
\hline & & 45 & & 0.22 & 0.35 & 0.25 & 0.32 & 0.27 & 0.33 \\
\hline & \multirow{4}{*}{ MAE } & 7 & 3.0 & 1.2 & 1.0 & 1.2 & 1.0 & 1.2 & 0.9 \\
\hline & & 15 & 2.8 & 1.1 & 0.9 & 1.1 & 0.9 & 1.0 & 0.9 \\
\hline & & 30 & & 1.2 & 0.9 & 1.0 & 1.1 & 1.0 & 0.8 \\
\hline & & 45 & & 1.2 & 1.1 & 1.1 & 1.1 & 1.0 & 1.0 \\
\hline
\end{tabular}

